\title{
Acompanhamento farmacoterapêutico dos pacientes portadores de Lúpus Eritematoso Sistêmico do município de Piripiri-PI
}

Pharmacotherapeutic follow-up of patients with Systemic Lupus Erythematus in the city of Piripiri-PI

Seguimiento farmacoterapéutico de pacientes con Lupus Eritemato Sistémico en la ciudad de Piripiri-PI

Ianna Paula Miranda Escórcio ORCID: https://orcid.org/0000-0003-4382-0028 Christus Faculdade do Piauí, Brasil

E-mail: iannapaulaescorcio@hotmail.com

Érida Kelly de Sousa Oliveira ORCID: https://orcid.org/0000-0002-9645-2137 Christus Faculdade do Piauí, Brasil E-mail: eridakelly@outlook.com

José Gabriel Fontenele Gomes ORCID: https://orcid.org/0000-0001-6114-0726 Christus Faculdade do Piauí, Brasil

E-mail: jgabrielfontenele@gmail.com

Daéri Karen de Sousa Oliveira ORCID: https://orcid.org/0000-0003-3091-2222 Christus Faculdade do Piauí, Brasil E-mail: daerikaren@gmail.com

Neirigelson Ferreira de Barros Leite ORCID: https://orcid.org/0000-0001-6505-1368 Christus Faculdade do Piauí, Brasil E-mail: neirigelson@hotmail.com

Guilherme Antônio Lopes de Oliveira ORCID: https://orcid.org/0000-0003-3820-0502 Christus Faculdade do Piauí, Brasil E-mail: guilhermelopes@live.com Mônica do Amaral Silva ORCID: https://orcid.org/0000-0001-6234-275X Christus Faculdade do Piauí, Brasil

E-mail: monica.amaral83@gmail.com

Gabriel Mauriz de Moura Rocha ORCID: https://orcid.org/0000-0003-1454-0414 Christus Faculdade do Piauí, Brasil E-mail: mauriz45@hotmail.com Genyvana Criscya Garcia Carvalho ORCID: https://orcid.org/0000-0002-8151-8746 Universidade Paulista, Brasil

E-mail: genyvanacarvalho@icloud.com

Luís Mário Rezende Júnior

ORCID: https://orcid.org/0000-0002-1145-9744

Christus Faculdade do Piauí, Brasil

E-mail: profmariorezende@gmail.com

\section{Resumo}

O presente estudo teve como objetivo realizar o acompanhamento farmacoterapêutico de pacientes portadores de LES do município de Piripiri-PI. O estudo trata-se de uma pesquisa de campo quantitativa, descritiva, longitudinal, observacional e intervencionista, com o emprego da técnica de observação direta em pacientes com LES, através do método Dáder de Acompanhamento Farmacoterapêutico. Para identificar o grau de adesão deste grupo de pacientes à medicação, adotou-se o Teste de Morisky, Green e Levine. Constatou-se, baseados na revisão da literatura realizada, que este é o primeiro estudo de pesquisa de campo quantitativo desenvolvido em um interior do estado do Piauí para avaliar a adesão à terapêutica medicamentosa em pacientes portadores de Lúpus Eritematoso Sistêmico. A pesquisa contou com dez participantes, todos residentes em Piripiri - Piauí, sendo $80 \%$ mulheres e $20 \%$ homens. No total, foram identificados 23 PRMs, com média de 2 a 3 para cada paciente. $20 \%$ dos pacientes apresentaram baixa 
adesão ao tratamento, $60 \%$ com adesão média e $20 \%$ com alta adesão. Foram identificadas $87,5 \%$ de interações medicamentosas moderadas, $6,25 \%$ leves e 6,25\% graves. Conclui-se que a adesão à terapêutica medicamentosa é um problema complexo e multifatorial e que o seguimento farmacoterapêutico é uma boa alternativa para avaliar a adesão e as RAMs associadas ao tratamento.

Palavras-chave: Lúpus eritematoso sistêmico; Cuidado farmacêutico; Interações medicamentosas.

\begin{abstract}
The present study aimed to carry out the pharmacotherapeutic follow-up of patients with SLE in the municipality of Piripiri-PI. The study is a quantitative, descriptive, longitudinal, observational and interventionist field research, using the direct observation technique in patients with SLE, using the Dáder Pharmacotherapeutic Monitoring method. To identify the degree of adherence of this group of patients to medication, the Morisky, Green and Levine test was adopted. Based on the review of the literature, it was found that this is the first quantitative field research study developed in the interior of the state of Piauí to assess adherence to drug therapy in patients with Systemic Lupus Erythematosus. The survey included ten participants, all of whom lived in Piripiri - Piauí, 80\% women and 20\% men. In total, 23 PRMs were identified, with an average of 2 to 3 for each patient. $20 \%$ of patients had low adherence to treatment, $60 \%$ with medium adherence and $20 \%$ with high adherence. $87.5 \%$ of moderate drug interactions, $6.25 \%$ mild and $6.25 \%$ severe were identified. It is concluded that adherence to drug therapy is a complex and multifactorial problem and that pharmacotherapeutic follow-up is a good alternative to assess adherence and ADRs associated with treatment.
\end{abstract}

Keywords: Systemic lupus erythematosus; Pharmaceutical care; Drug interactions.

\title{
Resumen
}

El presente estudio tuvo como objetivo realizar el seguimiento farmacoterapéutico de pacientes con LES en el municipio de Piripiri-PI. El estudio es una investigación de campo cuantitativa, descriptiva, longitudinal, observacional e intervencionista, utilizando la técnica de observación directa en pacientes con LES, utilizando el método de Monitoreo Farmacoterapéutico Dáder. Para identificar el grado de adherencia de este grupo de pacientes a la medicación se adoptó la prueba de Morisky, Green y Levine. Con base en la revisión de la literatura, se encontró que este es el primer estudio de investigación de campo cuantitativo desarrollado en el interior del estado de Piauí para evaluar la adherencia a la farmacoterapia en pacientes con Lupus Eritematoso Sistémico. La encuesta incluyó a diez participantes, todos los cuales vivían en Piripiri - Piauí, 80\% mujeres y 20\% hombres. En total, se identificaron 23 PRM, con un promedio de 2 a 3 por cada paciente. El $20 \%$ de los pacientes tuvo baja adherencia al tratamiento, el $60 \%$ con media adherencia y el $20 \%$ con alta adherencia. Se identificaron $87,5 \%$ de interacciones medicamentosas moderadas, $6,25 \%$ leves y $6,25 \%$ graves. Se concluye que la adherencia a la farmacoterapia es un problema complejo y multifactorial y que el seguimiento farmacoterapéutico es una buena alternativa para evaluar la adherencia y las RAM asociadas al tratamiento.

Palabras clave: Lupus eritematoso sistémico; Cuidado farmacéutico; Interacciones con la drogas.

\section{Introdução}

O organismo humano é capaz de produzir defesa através da resposta imune. Para tal, possui um sistema complexo denominado de imunológico que protege contra invasões estranhas ao corpo e que sejam, ou não, capazes de agredir o organismo. Porém, quando esse sistema apresenta falhas nesse processo de reconhecimento do não próprio, podem ser induzidas defesas imunológicas contra o próprio corpo (Prado et al., 2017).

Hipócrates (460 a 370 a.C) apresentou pela primeira vez as lesões do lúpus e, em 1851, as manifestações clínicas do lúpus sistêmico e cutâneo foram descritas por Cazenave. Em 1948, Hargraves e seus colegas descobriram uma célula específica no sangue de pacientes com lúpus sistêmico, que então passou a ser considerada como uma doença autoimune. E no ano de 1957, Friou observou a presença de anticorpos antinucleares, confirmando que o lúpus é na verdade um distúrbio do sistema imunológico do corpo (Sousa, Silva \& Partata, 2011).

Tal patologia trata-se de uma doença capaz de causar grande alteração no sistema imunológico humano e desencadear desordenadamente na produção de auto anticorpos. Tem como sua principal característica a produção de anticorpos antinuclear. É uma patologia que pode acometer o indivíduo em qualquer fase da vida, porém, aparece predominantemente entre os 16 e 55 anos. Quanto ao gênero, tem maior prevalência no sexo feminino (Costa et al., 2016).

O lúpus é classificado em três formas clinicas e é, possivelmente, provocado por alterações de fatores genéticos, hormonais ou ambientais (Costa \& Coimbra, 2014). Segundo o Protocolo Clínico e Diretrizes Terapêuticas- PCDT do Ministério da Saúde, os códigos da Classificação Internacional de Doenças - 10 (CID-10) para o Lúpus são o L93.0 para Lúpus discoide, 
L93.1 para o Lúpus cutâneo subagudo e M32.1 para identificar o Lúpus eritematoso sistêmico e M32.8 para outras formas de lúpus eritematoso disseminado (Brasil, 2013).

O LES pode evoluir com diversas lesões vasculares classificadas em trombóticas não inflamatórias e inflamatórias, reconhecendo-se, dentro das primeiras, a síndrome antifosfolipídica (SAF) e a púrpura trombocitopénica trombótica (PTT), e, dentro das segundas, a capilarite/vasculite lúpica e a leucotrombose. Esta última instala-se na sequência de uma síndrome de resposta inflamatória sistémica (SRIS) e caracteriza-se pela formação de rolhões de neutrófilos aderentes ao endotélio dos vasos de pequeno calibre. Pode corresponder a uma exacerbação da doença lúpica ou, então, a um processo de etiologia infecciosa (Costa, Santos \& Ferreira, 2003).

Essa é a forma clínica dos lúpus que apresenta alterações em vários sistemas orgânicos. Devido a essa característica é também a forma que apresenta maior complexidade. Possui etiologia multifatorial e indefinida com caráter inflamatório e crônico. Compromete geralmente órgãos internos, articulações e pele. Seu tratamento objetiva a preservação dos órgãos atingidos, controle da evolução da doença e diminuição do impacto causado pelas reações adversas da terapia medicamentosa utilizada (Galindo \& Veiga, 2010).

Essa forma apresenta polimorfismo clínico de reconhecimento tardio e uma variedade de sinais e sintomas comuns a outros quadros patológicos, motivo pelo qual há sempre uma evolução da doença até o reconhecimento do diagnóstico e início da terapêutica medicamentosa adequada (Prado et al., 2017). O Sistema Único de Saúde (SUS) atende os pacientes com LES através de assistência clínica e farmacológica. O profissional farmacêutico é atuante no componente especializado da assistência farmacêutica, responsável pela distribuição de medicamentos para LES. Cabe ao profissional farmacêutico estarem atualizados quanto à importância do acompanhamento farmacêutico em saúde, quais os principais fatores que levam a não adesão da terapia medicamentosa e quais as possíveis melhorias na farmacoterapêutica de pacientes com LES.

As manifestações clínicas dos lúpus estão intimamente ligadas à capacidade do organismo de resposta e correção dos efeitos deletérios causados. Por serem manifestações proteiformes, dependem da tipagem de anticorpos presentes, dos órgãos atingidos, e dos produtos celulares acometidos pela doença (Marnet \& Gonçalves, 2016).

Por atingir o organismo em vários órgãos e sistemas, a sintomatologia do lúpus é bastante variada. Devido a isso, essa patologia apresenta elevada complexidade no diagnóstico. O mesmo é feito a partir da suspeita da patologia e se dá por pesquisa específica e identificação de anticorpos e detecção de antígenos, sendo ainda necessário monitorar a atividade inflamatória devido a essa doença atingirem diferentes órgãos e tecidos simultaneamente (Sousa, Silva \& Partata, 2011).

Tem como queixas comuns a perda de peso, febre, fadiga e mal-estar. As alterações sistêmicas atingem com mais frequência às articulações, rins, pulmões, coração, sistema nervoso central, e pele. Essas manifestações clínicas podem ser recorrentes e permanecem constantemente ao longo da vida do portador de lúpus (Viana et al., 2010).

É necessário para a confirmação do diagnóstico que o paciente apresente além de manifestações clínicas, parâmetros imunológicos ou nefrite comprovada através de um exame de biopsia que demonstre a existência de auto anticorpos (Prado et al., 2017). Além disso, é necessário ainda o enquadramento da condição clínica em pelo menos quatro dos onze critérios descritos no quadro a seguir: 
Quadro 1 - Quadros Clínicos do Lúpus Eritematoso Sistêmico.

\begin{tabular}{|cc|}
\hline $\begin{array}{c}\text { MANIFESTAÇÕES } \\
\text { CLÍNICAS }\end{array}$ & CARACTERÍSTICAS \\
\hline Eritema fixo, plano ou elevado & Presente nas eminências malares, tendendo a poupar a região nasolabial \\
\hline Lesão discóide & $\begin{array}{r}\text { Lesão eritematosa infiltrada, com escamas queratóticas aderidas e tampões } \\
\text { foliculares, evoluindo com cicatriz atrófica e discromia }\end{array}$ \\
\hline Fotossensibilidade & Reação incomum e extrema da pele a raios solares ou iluminação artificial \\
\hline $\begin{array}{c}\text { Úlcera na cavidade oral ou } \\
\text { nasofaringe }\end{array}$ & Geralmente indolor \\
\hline Artrite & Não erosiva envolvendo duas ou mais articulações periféricas \\
\hline Alterações renais & Falência crônica e insidiosa do sistema renal \\
\hline Alterações neurológicas & Manifesta-se com a presença de convulsões ou psicose \\
\hline Alterações hematológicas & Alterações imunológicas e a presença de anticorpo antinuclear \\
\hline Alterações pulmonares & Hemorragia pulmonar, pneumonite agudalúpica \\
\hline Alterações endócrinas & Pode ocorrer alterações no ciclo menstrual na fase ativa da patologia \\
\hline Comprometimento vascular & Necrose de polpas, fenômeno Raynauld e feridas isquêmicas profundas \\
\hline
\end{tabular}

Fonte: Autores (2019).

O quadro acima expõe as manifestações que mais apontam para o diagnóstico do LES. Além dessas, a patologia ainda apresenta inúmeras outras condições. Estas acabam por propiciar a decisão de iniciar tratamentos diversos, uma vez que causam um diagnóstico difícil. O LES pode apresentar grandes diferenças ao se manifestar em um indivíduo e outro. Alguns mais discretos e em outros de forma abrupta, os quais necessitam de intervenções imediatas (Freire, Souto \& Ciconelli, 2011).

Usualmente, utiliza-se de análises laboratoriais para complementar o diagnóstico clínico. Os mesmos são requisitados para dar maior embasamento diagnóstico. Entre as análises laboratoriais, podem ser citados: a velocidade de hemossedimentação e a proteína $\mathrm{C}$ reativa, os quais são capazes de avaliar marcadores inespecíficos de quadros de inflação presente no Lúpus e também em casos de Infarto agudo do miocárdio (Rodrigues et al., 2017).

O hemograma é um importante exame laboratorial capaz de identificar alterações e, até denunciar a presença do Lúpus ativo. A análise da urina pode apresentar um marcador sensível a inflamações da bexiga ou renal, comum no lúpus, e devendo colaborar com os critérios de suspeitas para o diagnóstico. Proteinúria e hematúria são outras causas consideradas como importantes sinais. E por fim, e especificamente importante para nessa patologia, os testes de reação autoimunes para auto anticorpos que realizam uma triagem com grande importância clínica. Esta isola e marca presença de anticorpos antinuclear, fechando, juntamente com a clínica, o diagnóstico da LES (Brasil, 2013).

Segundo Sousa, Oliveira e Soares (2017), o tratamento da LES pode ser medicamentoso e/ou não-medicamentoso. O início do tratamento do LES já exige que o paciente faça melhoras nos seus hábitos e busque mudanças no estilo de vida, bem como se adeque a sua condição. O mesmo deve evitar exposição solar, cessar o tabagismo e o uso de álcool, se for o caso. Além também de passar a realizar atividade física regular e consumir uma alimentação balanceada.

No caso das mulheres, precauções em relação ao uso de anticoncepcionais devem ser tomadas, assim como profilaxia para verminoses, avaliação da sensibilidade a quadros alérgicos, prevenção de eventos tromboembolísticos, hipertensão, diabetes, dislipidemias, realização de sorologias para infecções sexualmente transmissíveis, e ainda prevenção de fatores que elevem o risco cardiovascular (Campos et al., 2013). 
Em relação à terapêutica medicamentosa do LES, Sousa, Silva e Partata (2011), afirmam que a mesma necessita ser individualizada e adequada a sintomatologia presente e as comorbidades existentes. É complexa, extensa e plausível tendo como principal foco o controle da resposta imuno-inflamatória e o cuidado para não evolução a quadros graves capazes de levar órgãos vitais à falência, como por exemplo, os rins.

Compondo os Protocolos Clínicos e Diretrizes Terapêuticas (PCDT), aprovado pela portaria 100/2013 traz em seu texto os tipos de tratamentos para a doença, tanto medicamentoso como não medicamentoso. Os fármacos são descritos e são utilizados de acordo com as alterações sistêmicas apresentadas pelos pacientes e a gravidade do quadro de cada uma dela. O tratamento medicamentos está descrito no quadro a seguir:

Quadro 2 - Tratamento das alterações sistêmicas do Lúpus Eritematoso Sistêmico.

\begin{tabular}{|cc|}
\hline FÁRMACO & APRESENTAÇãO \\
\hline Cloroquina & Comprimidos de $150 \mathrm{mg}$ \\
\hline Hidroxicloroquina & Comprimidos de $400 \mathrm{mg}$ \\
\hline Betametasona & Suspensão injetável de $(3 \mathrm{mg}+3 \mathrm{mg} / \mathrm{mL})$ \\
\hline Dexametasona & Comprimidos de $4 \mathrm{mg}$ \\
\hline Metilprednisolona & Pó para solução injetável de $500 \mathrm{mg}$ \\
\hline Prednisona & Comprimidos de 5 ou $20 \mathrm{mg}$ \\
\hline Azatioprina & Comprimidos de $50 \mathrm{mg}$ \\
\hline Cliclosporina & Cápsulas de $10,25,50,100$ mg e solução oral de $100 \mathrm{mg} / \mathrm{mL}-$ frasco de $50 \mathrm{~mL}$ \\
\hline Ciclofosfamida & Comprimidos de 50 mg e pó para solução injetável de $200 \mathrm{e} 1.000 \mathrm{mg}$ \\
\hline Danazol & Cápsulas de 100 ou $200 \mathrm{mg}$ \\
\hline Metotrexato & Comprimidos de 2,5 mg e solução injetável de $25 \mathrm{mg} / \mathrm{mL}$ \\
\hline Talidomida & Comprimido de $100 \mathrm{mg}$ \\
\hline
\end{tabular}

Fonte: Brasil (2013). Adaptado.

Os medicamentos do quadro são utilizados sempre de acordo com a situação clínica do paciente. Assim, não existe nenhum esquema pré-definido ou padronizado, visto a grande variedade de sintomas e modificações de um paciente para o outro. No entanto, em casos em que os pacientes se encontram sob controle, a retirada gradual de fármacos é feita. Caso ocorrer recidiva, o tratamento é reiniciado com a menor dose indicada do fármaco ou em associação com outros (Brasil, 2013).

Quanto à durabilidade do tratamento farmacoterapêutico, não existe um prazo pré-estabelecido. O mesmo será instituído baseado na progressão dos sintomas ou melhora, assim como nos critérios de normalidades que podem ser obtidos nos exames laboratoriais (Costa et al., 2016).

Para Reis e Costa (2010), dentre os problemas relacionados aos medicamentos (PRMs), pode-se destacar as interações, e as reações adversas, na terapia do LES. O metotrexato pode ocasionar o aumento de transaminases; a talidomida pode ter associação com o aparecimento de neuropatia periférica; e a Ciclosporina pode provocar insuficiência aguda, elevando os níveis de creatinina na corrente sanguínea.

Os medicamentos para o tratamento do LES estão disponíveis no sistema de saúde pública, com a dispensação feita através das farmácias excepcionais, dentro do componente especializado da assistência farmacêutica. São medicamentos em sua maioria de alto custo e que dependem de continuidade de estoque, pois a interrupção do tratamento pode levar o paciente a regredir, ter piora clínica e até chegar ao óbito (Sousa, Oliveira \& Soares, 2017). 
Segundo Fritzen, Matter e Paniz (2017) a adesão de medicamentos essências varia de 15.6 a 76,8\% e que é fundamental o acesso regular ao medicamento em tempo hábil e sem faltas, para que haja a resposta possível da recuperação do paciente. Vários fatores influenciam a continuidade de um tratamento, que vai desde a aceitação e o comprometimento do indivíduo até a disponibilidade do medicamento de forma acessível. Dentro deste contexto, o Componente Especializado da Assistência Farmacêutica (CEAF) destina-se atualmente à aquisição e dispensação de medicamentos de alto custo e a medicamentos que tratam a doença crônica que necessita de tratamento ambulatorial.

Segundo Farias e Martins (2013), as dúvidas dos pacientes devem ser sanadas, como uma importante estratégia para o sucesso do tratamento, nesse contexto, entra a assistência farmacêutica clínica, assim, a conscientização do paciente sobre as inúmeras alterações sistêmicas que a doença pode causar se faz importante. O farmacêutico é capaz de atuar promovendo entendimento a respeito da terapia medicamentosa e visa a colaboração dos pacientes e seus domiciliares e um eficaz autocuidado.

O método Dáder, criado pelo "Grupo de Investigación en Atención Farmacéutica de la Universidad de Granada", foi desenvolvido para fornecer ao profissional farmacêutico algumas ferramentas e um conjunto de abordagens e procedimentos para a realização do SF. Ele se destaca por ser um procedimento operativo simples e adaptável que permite a realização do SF de forma sistematizada e documentada. Com as informações obtidas dos pacientes é possível traçar um retrato da saúde e tratamento do paciente em momentos distintos, assim como avaliar os resultados da farmacoterapia (Dáder, Hernández \& Castro, 2009).

O método Dáder se distingue por melhorar e adequar-se às necessidades de uma atividade clínica - o SF, que está em contínuo desenvolvimento. Isto quer dizer que o método tende a sofrer reformas ou inovações (atualizações) devidas à experiência adquirida com a prática do SF. Ele começa pelo oferecimento do serviço ao paciente pelo profissional farmacêutico; caso seja aceito, é marcada a primeira entrevista para a coleta de dados do doente e abertura de sua história farmacoterapêutica. Adiante é realizada a avaliação do estado de situação com o objetivo de relacionar os problemas de saúde e o uso de medicamentos relatados pelo paciente e, com isso, identificar possíveis PRMs, sendo feito planos de intervenções farmacêuticas para resolver os mesmos. Logo após, é realizada a avaliação dos resultados obtidos com essas intervenções, bem como entrevistas para avaliação das mudanças nos problemas de saúde do paciente (Dáder, Hernández \& Castro, 2009).

Diversas estratégias surgiram para auxiliar na investigação da adesão ao uso de medicamentos. Neste sentido, Morisky e Green apresentam uma escala de auto-relato, teste de Morisky e Green, composta de quatro perguntas para identificar atitudes e comportamentos frente à tomada de remédios, que têm se mostrado úteis para identificação de pacientes aderentes ou não ao tratamento. Entender a adesão dos pacientes às medicações é importante para atingir os resultados clínicos planejados e, ainda, diminuir os gastos médico-hospitalares. Assim, estudos que proporcionam esse acompanhamento aos pacientes com LES são relevantes para demonstrar a importância da prevenção e resolução de problemas relacionados ao medicamento, assim como ações para evitar, ou pelo menos diminuir, o abandono da terapia.

\section{Metodologia}

O estudo trata-se de uma pesquisa de campo quantitativa, descritiva, longitudinal, observacional e intervencionista, com o emprego da técnica de observação direta em pacientes com LES, cujo código na Classificação Internacional de Doenças (CID), é M32.1 (Ferreira, 2015). O método utilizado para o acompanhamento farmacoterapêutico foi fundamentado no Método Dáder. De acordo com Santos (2016), o Método Dáder foi elaborado para o âmbito da farmácia comunitária, tendo sofrido adaptações para diferentes setores assistenciais, grupos populacionais e países. De forma geral, o método é centrado em três aspectos essenciais, sendo: análise global (avaliação do paciente em relação aos problemas de saúde e medicamentos utilizados), 
plano de ação (execução de intervenções com o paciente para resolver e/ou prevenir problemas na farmacoterapia) e controle e avaliação do processo (farmacêutico e paciente realizam avaliações periódicas se os objetivos planejados foram alcançados).

Para identificar o grau de adesão deste grupo de pacientes à medicação, adotou-se o Teste de Morisky, Green e Levine de quatro questões. O estudo foi realizado no componente especializado da assistência farmacêutica, ligada à III Coordenação Regional de Saúde, na cidade de Piripiri-PI. A coleta de dados foi obtida por meio de entrevista com os pacientes que aceitaram, tendo compreendido e assinado o TCLE. Para tanto, foi utilizado um questionário estruturado.

Os critérios de inclusão foram todos os pacientes de ambos os sexos que independentemente da idade, apresentavam LES e recebiam o medicamento no período de realização da pesquisa e, que aceitaram ser entrevistados tendo compreendido e assinado o Termo de Consentimento Livre e Esclarecido (TCLE). Os critérios de exclusão foram aqueles pacientes que se negaram a participar ou não concordaram em assinar o termo de Consentimento Livre e Esclarecido, e aqueles que se negaram a responder os formulários propostos.

Foram realizadas entrevistas sucessivas com aplicação de formulários específicos para obtenção de informações. A metodologia preconizada para realização da pesquisa foi fundamentada no método Dáder que compreende a 5 etapas.

$1^{\text {a }}$ etapa: oferta de serviço, na qual busca explanar, com clareza e concisão, a prestação de cuidado a ser recebida. Há alguns aspectos que precisam ficar determinados na oferta do serviço, tais como que o objetivo do Seguimento Farmacoterapêutico (SF) é obter o máximo de benefícios dos medicamentos utilizados.

$2^{\mathrm{a}}$ etapa: primeira entrevista Farmacêutica. Essa etapa constituiu a principal fonte de informação para o Seguimento Farmacoterapêutico (SF). Os pacientes foram estimulados a relatar seus problemas de saúde ou qualquer aflição relacionada ou não ao uso de medicamentos.

$3^{\text {a }}$ etapa: fase de estudo. Essa etapa serviu para avaliar a farmacoterapia do paciente e visualizar seu estado geral de saúde. Foram realizadas pesquisas de informações em bases científicas confiáveis para obtenção de informações objetivas sobre os problemas de saúde e da medicação do paciente.

$4^{\mathrm{a}}$ etapa: fase de avaliação. O objetivo dessa fase foi identificar os Resultados Negativos associados à Medicação (RNMs) que o paciente apresentou ou pôde vir a apresentar durante a pesquisa, a fim de levantar intervenções farmacêuticas para a resolução dos mesmos.

$5^{\mathrm{a}}$ etapa: fase de Intervenção ou $2^{\mathrm{a}}$ entrevista, foi elaborado o plano de atuação com o paciente, o qual é um programa de trabalho continuado que contém os registros das diferentes intervenções que se deve realizar para melhorar ou preservar o estado de saúde do paciente. Neste segundo encontro, foi atualizado o Estado de Situação do paciente, exposto o Plano de Atuação e realizado as intervenções farmacêuticas necessárias. Por fim, foi marcado uma terceira entrevista para se analisar os resultados das intervenções propostas, assim como aplicação de outras intervenções que foram ser necessárias. O projeto de pesquisa foi aprovado pelo Comitê de Ética em Pesquisa do Hospital Getúlio Vargas sob o protocolo Nº 10993019.7.0000.561, por estar de acordo com os princípios éticos. Os entrevistados foram informados a respeito da intenção e dos objetivos do estudo.

\section{Resultados e Discussão}

\subsection{Caracterização sociodemográfica}

Constatou-se, baseados na revisão da literatura realizada, que este é o primeiro estudo de pesquisa de campo quantitativo desenvolvido em um interior do estado do Piauí para avaliar a adesão à terapêutica medicamentosa em pacientes portadores de Lúpus Eritematoso Sistêmico. Foram dez os participantes da pesquisa e todos residentes na cidade de Piripiri. A descrição completa das características sociodemográficas encontra-se no Quadro 3. 
Quadro 3 - Características sociodemográficas dos pacientes portadores de LES no município de Piripiri-PI.

\begin{tabular}{|c|c|c|}
\hline $\begin{array}{c}\text { Características } \\
\text { Sociodemográficas }\end{array}$ & Frequência Absoluta (n) & Frequência Relativa (\%) \\
\hline \multicolumn{3}{|l|}{ SEXO } \\
\hline Masculino & 8 & $80 \%$ \\
\hline Feminino & 2 & $20 \%$ \\
\hline \multicolumn{3}{|l|}{ ESTADO CIVIL } \\
\hline Solteiro & 2 & $20 \%$ \\
\hline Casado & 8 & $80 \%$ \\
\hline \multicolumn{3}{|l|}{ ESCOLARIDADE } \\
\hline Ensino Fundamental Completo & 3 & $30 \%$ \\
\hline Ensino Médio Completo & 4 & $40 \%$ \\
\hline Ensino Superior Completo & 3 & $30 \%$ \\
\hline \multicolumn{3}{|l|}{ IDADE } \\
\hline $27-37$ anos & 5 & $50 \%$ \\
\hline $38-48$ anos & 3 & $30 \%$ \\
\hline $49-59$ anos & 2 & $20 \%$ \\
\hline \multicolumn{3}{|l|}{ COM QUEM MORA? } \\
\hline Sozinho & 2 & $20 \%$ \\
\hline Família & 8 & $80 \%$ \\
\hline \multicolumn{3}{|l|}{ ETNIA } \\
\hline Branco & 4 & $40 \%$ \\
\hline Negro & 6 & $60 \%$ \\
\hline
\end{tabular}

Fonte: Autores (2019).

Com relação ao sexo, no presente estudo, $80 \%$ dos pacientes eram mulheres e $20 \%$ homens (Quadro 1). Esse resultado é condizente com o estudo de Conde et al. (2009), o qual apresentou predomínio de acometimento do sexo feminino (95\%), numa proporção de 20 mulheres para cada homem. Corroborando com isso, o estudo de Madureira et al., apresentou 84\% das pacientes sendo do sexo feminino. Segundo Costa; Coimbra, (2014), este predomínio pode ser justificado por uma provável ação dos hormônios femininos. Sem se descartar ainda um possível efeito protetor dos hormônios masculinos ou relacionado ao cromossomo X.

Outra característica social que, segundo Barreto et al. (2014), influencia na não adesão à farmacoterapia é o estado civil. Os indivíduos com LES vivenciam limitações no estilo de vida e tem que se adaptar às novas formas de conduzir suas rotinas. Isso acarreta, por vezes, frustrações e insatisfação. Desta maneira, o envolvimento de um conjugue ou membro familiar no tratamento, torna-se componente facilitador para a adesão à terapia medicamentosa por representar apoio emocional.

Outrossim, Araújo e Garcia (2006), apontam o estado civil como uma variável sociodemográfica preditora da adesão ao tratamento, sendo que, quando comparados aos solteiros, os portadores de LES casados apresentaram chance duas vezes maior de realizar adequadamente o tratamento. No estudo realizado, 20\% dos pacientes apresentaram-se solteiros, no qual um destes mora sozinho, fator que pode contribuir para uma baixa adesão. Já o outro paciente, mora com os pais, que pode demonstrar maior cuidado e apoio familiar, o que facilitaria uma melhor adesão ao tratamento (alta adesão). Dessa forma, esse 
fato confirma o que diz Moreira (2005), no tocante ao apoio familiar estar fortemente associado a adesão, fator este de grande importância para a ATM em doenças crônicas. Assim, pessoas que estão isoladas, distantes do convívio familiar e social, podem apresentar maiores chances de não aderir ao tratamento.

Vale ressaltar ainda que, $80 \%$ dos pacientes são casados, porém nem todos eles apresentam uma alta adesão conforme explícito na literatura, situação que é ocasionada por diversos fatores. Dentre esses fatores, o que se mostra comum a todos os pacientes é a participação ativa dessas pessoas na composição da renda familiar. Dessa forma, a ocupação tem relação direta com os descuidos no tratamento, no qual os motivos podem ser tanto pelo esquecimento de tomar a medicação, quanto pela desatenção com os horários.

O grau de escolaridade é um importante indicador social exercendo influência, também, sobre as oportunidades de emprego e de salários e, nesta medida, condiciona o poder aquisitivo das famílias. A escolaridade esteve associada com a Adesão à Terapia Medicamentosa (ATM) na presente pesquisa, corroborando com outros estudos; Barreto et al. (2014) também identificaram o baixo grau de escolaridade como significante fator que leva a não adesão no LES. A baixa escolaridade associase também com a dificuldade em compreender as recomendações dos profissionais de saúde e a importância do uso contínuo dos medicamentos para o controle da patologia, por isso caracteriza-se como um fator socioeconômico influente da não adesão ao tratamento. No caso do LES, que é uma condição crônica assintomática, a baixa escolaridade constitui ainda um agravante por dificultar o reconhecimento da necessidade de assistência médica e continuidade do tratamento seja ele medicamentoso ou não.

Na pesquisa, o ensino médio completo foi o nível de instrução prevalente, representado por $40 \%$ dos pacientes, no qual faz com que não exista uma baixa adesão totalmente, mas sendo representado por uma média adesão, segundo o teste de Morisky, Green e Levine. Em contrapartida, 30\% apresentaram ensino superior completo, representando um total de melhor adesão ao tratamento, pois dois desses pacientes possuíram alta adesão, o que confirma o exposto na literatura. Já o terceiro paciente apresentou média adesão, pois apesar de possuir boa escolaridade, sofre com sintomas de depressão, o que influencia negativamente para a eficácia do tratamento (Cabrera, 2016).

O LES pode ocorrer em qualquer idade, mas é mais frequente entre os 20 e 45 anos, com maior incidência próxima aos 30 anos, ou seja, em mulheres jovens na fase reprodutiva, segundo Conde (2009). No presente estudo, 50\% dos pacientes têm idade média entre 27 a 37 anos; $30 \%$ têm entre 38 a 48 anos e $20 \%$ entre 49 a 59 anos, corroborando com o que se diz na literatura. Sendo que nesses pacientes, a média de convívio com a doença após a comprovação de diagnóstico está na média de 10,4 anos. O LES pode ocorrer em todas as etnias, no entanto, a doença é mais frequente em negros que em brancos. A incidência anual nos EUA é de 27,5/milhão para mulheres brancas e 75,4/milhão para mulheres negras. O resultado encontrado no presente estudo é condizente com essa estimativa, na qual a prevalência se dá em $60 \%$ para pacientes negros (sendo 5 mulheres e 1 homem) e $40 \%$ para pacientes brancos (sendo 3 mulheres e 1 homem).

\subsection{Descrição de Problemas Relacionados a Medicamentos}

Os problemas relacionados a medicamentos (PRM's) são todas as situações, que durante o processo de utilização dos medicamentos, podem causar o aparecimento de um resultado negativo associado à medicação. Seja as reações adversas por medicamentos; a não adesão ao tratamento e interações medicamentosas (Jacomini \& Silva, 2011). O profissional farmacêutico, ao identificar e classificar um PRM, deve intervir na farmacoterapia, contribuindo para uma melhora na adesão medicamentosa e na qualidade de vida dos usuários de medicamentos (Meneses et al., 2010).

No Brasil, o instrumento de AFT mais utilizado é o método Dáder, o qual foi desenvolvido pelo grupo de Investigação em Atenção Farmacêutica da Universidade de Granada, na Espanha, e baseia-se na obtenção da história farmacoterapêutica do paciente e na avaliação de seu estado de situação em uma data determinada. Essa metodologia tem o objetivo de identificar e resolver os possíveis PRMs apresentados (Brune et al., 2014). 
De acordo com a metodologia Dáder, a farmacoterapia atende os critérios de necessidade, efetividade e segurança em seu uso, que também servem para a detecção de PRMs. O PRM de necessidade, ou PRM 1, acontece quando o paciente não toma os medicamentos de que necessita, enquanto o PRM 2 acontece quando o paciente toma medicamentos que não necessita. Quanto à efetividade, divide-se em PRM 3, que é quando o paciente toma um medicamento que estando indicado para sua situação, mas está mal selecionado; e em PRM 4, quando o paciente toma uma dose inferior do medicamento indicado para sua situação. E por último, quanto à segurança, sendo dividido em PRM 5, que é quando o paciente toma dose superior do medicamento indicado para sua situação e em PRM 6, no qual o paciente toma um medicamento que provoca uma reação adversa (Zanella \& Assini, 2008).

Tais PRM's podem ser identificados em qualquer uma das parcelas de população que fazem uso de medicamento e foi determinante para descrever os possíveis PRM's dos pacientes portadores de LES. Com isso, no Gráfico 1 está descrito o percentual dos PRM's analisados diante dos 10 pacientes avaliados, visto que um único paciente pode apresentar mais de um PRM.

Gráfico 1 - Análise de percentual de PRMs dos pacientes entrevistados.

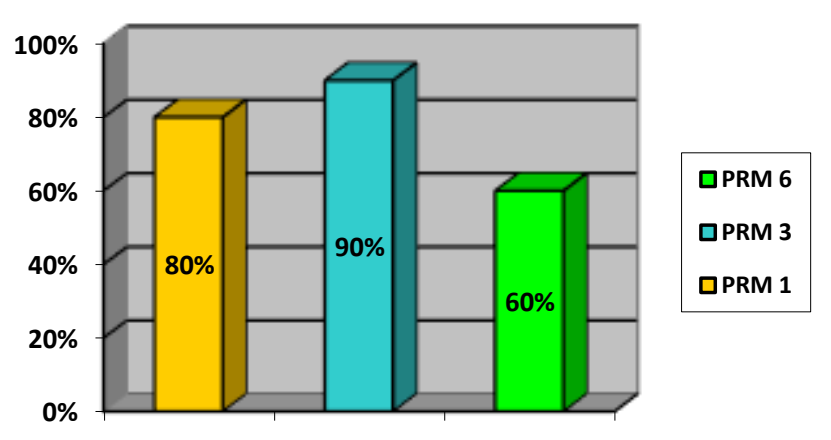

Fonte: Autores (2019).

Foram identificados 23 PRM em um total de 10 pacientes, no qual cada um dos pacientes apresentava em média de 2 a 3 PRM. Segundo a classificação proposta pelo Segundo Consenso de Granada, os PRM mais prevalentes neste estudo foram 80\% do PRM 1 (o paciente não toma os medicamentos de que necessita), 90\% do PRM 3 (o paciente toma um medicamento, que estando indicado para sua situação, está mal selecionado) e 60\% do PRM 6 (o paciente toma um medicamento que provoca uma reação adversa), os quais relacionam-se à necessidade, efetividade e a segurança no uso dos medicamentos, respectivamente. O PRM 2 (o paciente toma medicamentos que não necessita), 4 (o paciente toma uma dose inferior do medicamento indicado para sua situação) e o PRM 5 (o paciente toma dose superior do medicamento indicado para sua situação) não foram encontrados nesse estudo.

\subsection{Fatores relacionados à Adesão à Terapêutica Medicamentosa}

Os dados foram analisados e identificados quanto ao grau de adesão por meio do Teste de Morisky, Green e Levine de quatro questões. Este teste além de aferir o grau de adesão (em baixa, média e alta adesão), também define a intencionalidade da não adesão, em intencional ou não intencional. É intencional quando os pacientes respondem sim para a $3^{\mathrm{a}} \mathrm{e} / \mathrm{ou}$ a $4^{\mathrm{a}}$ pergunta do teste. E não intencional, quando respondem não à $3^{\mathrm{a}}$ e/ou à $4^{\mathrm{a}}$ pergunta. Dos 8 pacientes que não tiveram adesão, $20 \%$ responderam sim e $80 \%$ responderam não à $3^{\mathrm{a}} \mathrm{e}$ a $4^{\mathrm{a}}$ pergunta. 
A partir da análise dos dados, a prevalência quanto ao grau de adesão em baixa adesão foi de 20\%; de média adesão 60\% e de alta adesão 20\% dos 10 pacientes entrevistados em relação às 4 questões do Teste de Morisky, Green e Levine, no qual tais perguntas estão descritas no Quadro 4. As respostas obtidas para cada pergunta sobre o teste de adesão foram examinadas individualmente e encontram-se descritas no Quadro 4 o percentual de respostas positivas e o de respostas negativas. De acordo com os dados obtidos, verificou-se que a razão mais citada para a não adesão foi a desatenção com os horários de administração dos medicamentos $(80 \%, \mathrm{n}=8)$ seguida pelo esquecimento $(80 \%, \mathrm{n}=8)$.

Quadro 4 - Frequência de respostas positivas e negativas às questões do teste de Morisky, Green e Levine.

\begin{tabular}{|c|c|c|}
\hline \multicolumn{3}{|l|}{ FREQUÊNCIA RELATIVA } \\
\hline QUESTÕES & SIM & NÃO \\
\hline A Sr ${ }^{\mathrm{a}}$ se esquece de tomar os seus remédios? & $80 \%$ & $20 \%$ \\
\hline A $\mathrm{Sr}^{\mathrm{a}}$ costuma tomar seus remédios fora do horário? & $80 \%$ & $20 \%$ \\
\hline Quando a Sra está se sentindo melhor, para de tomar seus remédios? & $20 \%$ & $80 \%$ \\
\hline Às vezes, se a $\mathrm{Sr}^{\mathrm{a}}$ se sente pior tomando seus remédios, para de toma-los? & $20 \%$ & $80 \%$ \\
\hline
\end{tabular}

Fonte: Autores (2019).

De acordo com o teste de Morisky, o uso inadequado de medicamentos ocorre em uma ou em todas as seguintes formas: esquecimento, falta de cuidado, interromper o medicamento quando sentir-se melhor ou interromper o medicamento quando sentir-se pior. É de fácil medida, validado, com um número relativamente pequeno de questões compreensíveis, que proporcionam a verificação da atitude do usuário frente à tomada de medicamentos (Borges et al., 2011).

No entanto, o Projeto Adesão da Organização Mundial da Saúde (OMS), tem como definição de adesão a tratamentos crônicos, uma junção de outras duas definições de Haynes e Rand, que conceituam adesão como o grau em que o comportamento de uma pessoa representado pela ingestão de medicação, o acompanhamento da dieta, as alterações no estilo de vida correspondem e concordam com as recomendações de um profissional de saúde. Já Rocha et al. (2008) descrevem adesão ao tratamento como um meio para se alcançar um fim, um tratamento para a manutenção ou melhora da saúde, visando reduzir os sinais e sintomas de uma doença (Cardoso, 2011).

O não reconhecimento do LES como uma doença crônica se dá em $40 \%$ dos pacientes entrevistados, seja pela negação da patologia ou pelo desconhecimento sobre sua cronicidade. Por isso se faz necessário uma intervenção farmacêutica. Segundo Santos (2009) esclarecer sobre o tratamento do paciente, bem como proporcionar informações claras no intuito de orientar sobre o que está acontecendo com seu organismo, são fundamentais para a conscientização do portador de LES e aumento da sua adesão ao tratamento.

\subsection{Reações Adversas aos medicamentos}

Todos os pacientes entrevistados faziam o uso da terapia medicamentosa para o tratamento da LES e retiravam os medicamentos via Sistema Único de Saúde (SUS) por prescrição médica, por meio da farmácia excepcional da cidade de Piripiri. Todos eles relataram algumas reações adversas, no qual a dor de cabeça foi predominante (60\%), seguido por fadiga (40\%) e náuseas (30\%), estando descrito os dados no Quadro 5. 
Quadro 5 - Percentual de RAMs dos pacientes portadores de LES.

\begin{tabular}{|c|c|}
\hline REAÇÕES ADVERSAS & VARIÁVEIS N(\%) \\
\hline Azia & $1(10 \%)$ \\
\hline Boca seca & $1(10 \%)$ \\
\hline Catarata Subcapsular Posterior & $1(10 \%)$ \\
\hline Convulsão & $1(10 \%)$ \\
\hline Desconforto Abdominal & $1(10 \%)$ \\
\hline Osteoporose & $1(10 \%)$ \\
\hline Pressão Alta & $1(10 \%)$ \\
\hline Sonolência & $1(10 \%)$ \\
\hline Trombocitopenia & $1(10 \%)$ \\
\hline Náusea & $3(30 \%)$ \\
\hline Visão borrada & $3(30 \%)$ \\
\hline Fadiga & $4(40 \%)$ \\
\hline Dor de cabeça & $6(60 \%)$ \\
\hline
\end{tabular}

Fonte: Autores (2019).

As Reações Adversas a Medicamentos (RAM) são definidas como qualquer resposta prejudicial ou indesejável e não intencional que ocorre com medicamentos em doses usualmente empregadas no homem para profilaxia, diagnóstico e tratamento de doença. As RAMs figuram a quinta causa de mortes nos Estados Unidos, e no Brasil os estudos sobre o tema ainda são escassos. Inúmeros fatores podem influenciar no aparecimento de RAM, tais como idade, sexo, gênero, comorbidades e uso concomitante de vários medicamentos, entre outros. As RAMs estão associadas com a não adesão aos pacientes portadores de LES que utilizam vários medicamentos para sua patologia. É importante salientar que o paciente deve receber informações com respeito à ocorrência de prováveis efeitos adversos ao tratamento recebido e de como se portar frente ao surgimento dos mesmos.

A presença de RAM ocorreu em percentual considerável dos pacientes e a prednisona (da classe dos glicocorticosteroides) foi apontada como o principal medicamento causador das mesmas. Dos portadores de LES, 70\% fazem o uso dessa medicação e destes, $60 \%$ sentem dores de cabeça. A prednisona causa também alteração nos ossos e nos músculos, o que ocasionou a perda de massa óssea e provocou osteoporose na paciente A, que faz uso da medicação desde a descoberta da doença (há 9 anos). Por isso o fato de a mesma utilizar o cálcio, a fim de combater os efeitos adversos do glicocorticóide. Já o paciente B, tem como reação adversa a convulsão, portanto, faz o uso da Fenitoína para o seu controle, sendo que esta droga causou outra reação adversa, a trombocitopenia (diminuição no número de plaquetas sanguíneas). O paciente C apresentou catarata subscapular posterior como um efeito indesejável ainda à prednisona. Ele fazia o uso da medicação de $80 \mathrm{mg}$, e como houve sequelas para sua visão, foi diminuída sua dose para $5 \mathrm{mg}$. Já o paciente D apresentou como reação adversa um aumento na sua pressão arterial, causando arritmia cardíaca devido a alteração estrutural e elétrica do músculo cardíaco.

Tem-se demonstrado que mais de um terço dos indivíduos tratados com Glicocorticóides por 5 a 10 anos terá uma fratura por osteoporose como efeito adverso. O paciente pode permanecer assintomático por vários anos, e a perda óssea ocorre muito antes de outros sinais evidentes como atrofia cutânea, equimoses, perda muscular proximal. No entanto, estudos têm mostrado que menos de 50\% dos pacientes em tratamento com GC em longo prazo são avaliados em relação à osteoporose, e menos de $25 \%$ são tratados. Este efeito ocorre por diminuição do número e da atividade dos osteoblastos e aumento da função dos osteoclastos. Os ossos mais afetados, sendo assim os mais sujeitos a fraturas, são os que têm maior conteúdo trabecular, 
como as costelas, corpos vertebrais e ossos pélvicos. A fratura de quadril é a mais grave daquelas provocadas pela osteoporose (Pereira et al., 2007).

A hidroxicloroquina (da classe dos antimaláricos) também prevalece na terapia de $70 \%$ dos pacientes entrevistados, sendo responsável por ocasionar como RAM, a visão borrada/turva, em 30\% destes. A Azatioprina (da classe dos imunossupressores) está presente na terapia de $20 \%$ dos pacientes, fazendo-os relatar como efeitos adversos vômitos, náuseas e sonolência. O metotrexato (da classe antineoplásica) se faz presente na terapia de $20 \%$ dos pacientes entrevistados, os quais relataram sentir desconforto abdominal e fadiga excessiva. A talidomida (sedativo), indicada na terapia para LES, e utilizada por um único paciente, faz o relatar como RAM muita sonolência.

Os fármacos para a terapêutica específica do LES mais utilizados na pesquisa foram os glicocorticoides seguidos dos antimaláricos, imunossupressores e antineoplásicos. É evidente que uma enfermidade que pode acometer múltiplos órgãos e sistemas com diferentes graus de atividade apresente tratamento diversificado. No entanto, a avaliação do uso de medicamentos para o tratamento das comorbidades presentes no LES, tais como a hipertensão arterial sistêmica e dislipidemias, entre outras, infelizmente não tem recebido a devida atenção. A maioria dos estudos que avaliam o tratamento do paciente com LES o fazem somente sobre a ótica da doença de base (Santos, 2009).

Entretanto, a terapêutica medicamentosa deve ser avaliada no seu conjunto. O paciente, além dos medicamentos específicos para o LES, deve aderir a outros fármacos para comorbidades do tratamento em sua totalidade. Telles et al. (2007) discutem este aspecto, pois ao verificarem as razões de risco para enfermidade coronariana em pacientes com LES assistidos em um trabalho de reumatologia no estado de Minas Gerais, observaram que, apesar da dislipidemia ser o terceiro fator de risco mais frequente na população estudada, o tratamento estava sendo inadequado. De acordo com os autores, as prováveis causas para este acontecimento seriam os cuidados médicos com aspectos considerados mais relevantes no tratamento do Lúpus, o empecilho financeiro dos pacientes para compra de fármacos e a volumosa quantidade e diversidade de medicamentos necessários pelos indivíduos portadores de lúpus, acabando-se por priorizar o uso de certos medicamentos.

A viabilidade de o medicamento provocar efeitos adversos acaba sendo, na maioria das vezes, omitida pelo prescritor, receoso de que as informações negativas relativas aos medicamentos possam prejudicar a adesão do paciente ao tratamento ou mesmo que, por autosugestão, o paciente venha a sentir o efeito adverso comentado. Manter o medicamento, mesmo sentindo algum sintoma negativo durante o uso dos medicamentos, aumentou em $80 \%$ a chance de adesão naqueles que continuam tomando o medicamento em comparação com aqueles que o suspendem. Barreto (2014) identificou a RAM como sendo um fator determinante para a adesão. Dos pacientes entrevistados, todos eles relataram a presença de RAM.

\subsection{Interações medicamentosas relacionadas ao LES}

Quadro 6 - Principais interações medicamentosas para o tratamento de LES.

\begin{tabular}{|c|c|c|c|c|}
\hline $\begin{array}{c}\text { MEDICAMENTO } \\
\text { DA TERAPIA }\end{array}$ & $\begin{array}{c}\text { MEDICAMENTO } \\
\text { EXTERNO À } \\
\text { TERAPIA }\end{array}$ & $\begin{array}{c}\text { GRAU DE } \\
\text { INTERAÇÃo }\end{array}$ & CFEITO CLÍNICO & CONDUTA \\
\hline Azatioprina & Àcido Acetilsalić́lico & Moderado & Maior risco hemorrágico & $\begin{array}{c}\text { Monitoração do tempo de } \\
\text { protombina; monitorar } \\
\text { redução dos efeitos } \\
\text { terapêuticos do Ácido } \\
\end{array}$ \\
& & & & Acetilsalicílico \\
\hline
\end{tabular}


Research, Society and Development, v. 10, n. 4, e33110413482, 2021

(CC BY 4.0) | ISSN 2525-3409 | DOI: http://dx.doi.org/10.33448/rsd-v10i4.13482

\begin{tabular}{|c|c|c|c|c|}
\hline Azatioprina & Captopril/Enalapril & Moderado & $\begin{array}{c}\text { Maior efeito terapêutico } \\
\text { da Azatioprina }\end{array}$ & $\begin{array}{l}\text { Monitorar aumento dos } \\
\text { efeitos tóxicos da Azatioprina } \\
\text { (Neutropenia) }\end{array}$ \\
\hline Prednisona & Àcido Acetilsalicílico & Moderado & $\begin{array}{l}\text { Maiores ulcerações e } \\
\text { sangramentos } \\
\text { gastrintestinais; menor } \\
\text { filtração glomerular e } \\
\text { aumento do metabolismo } \\
\text { do ácido acetilsalicílico }\end{array}$ & $\begin{array}{l}\text { Monitorar terapia e reduzir } \\
\text { gradativamente a dose do } \\
\text { Ácido Acetilsalicílico } \\
\text { juntamente com a Prednisona }\end{array}$ \\
\hline Prednisona & Ibuprofeno & Moderado & $\begin{array}{c}\text { Aumenta o risco de } \\
\text { efeitos colaterais no trato } \\
\text { gastrintestinal, como } \\
\text { inflamações, } \\
\text { sangramentos, ulcerações } \\
\text { e raramente perfuração }\end{array}$ & $\begin{array}{c}\text { Diminuição da dose do } \\
\text { Ibuprofeno; Incluir na terapia } \\
\text { um protetor gástrico ou } \\
\text { substituir o antiinflamatório } \\
\text { não esteroidal }\end{array}$ \\
\hline Prednisona & Metformina & Moderado & $\begin{array}{l}\text { Interfere no controle da } \\
\text { glicose sanguínea e reduz } \\
\text { a eficácia da Metformina }\end{array}$ & $\begin{array}{c}\text { Monitorar os níveis de } \\
\text { glicose sanguínea. É } \\
\text { necessário um ajuste na dose } \\
\text { dos medicamentos para } \\
\text { diabetes durante e após o } \\
\text { tratamento com Prednisona }\end{array}$ \\
\hline Prednisona & Hidroclorotiazida & Moderado & $\begin{array}{l}\text { Causa dores e cãibras } \\
\text { musculares, perda de } \\
\text { apetite, fraqueza, tontura } \\
\text { e confusão }\end{array}$ & $\begin{array}{l}\text { Necessário ajuste de dose ou } \\
\text { testes especiais para o uso } \\
\text { com segurança dos dois } \\
\text { medicamentos }\end{array}$ \\
\hline Prednisona & Enalapril & Moderado & $\begin{array}{l}\text { Prednisona reduz os } \\
\text { efeitos do Enalapril na } \\
\text { redução da pressão } \\
\text { arterial, além de causar } \\
\text { retenção de sódio e água } \\
\text { com uso prolongado de } \\
\text { Prednisona }\end{array}$ & $\begin{array}{l}\text { Necessário ajuste da dose ou } \\
\text { frequente monitoramento da } \\
\text { terapia }\end{array}$ \\
\hline Prednisona & Anlodipino & Moderado & $\begin{array}{l}\text { Prednisona reduz os } \\
\text { efeitos do Anlodipino na } \\
\text { redução da pressão } \\
\text { arterial, além de causar } \\
\text { retenção de sódio e água } \\
\text { quando o uso da } \\
\text { Prednisona é prolongado }\end{array}$ & $\begin{array}{l}\text { Necessário ajuste da dose ou } \\
\text { frequente monitoramento da } \\
\text { terapia }\end{array}$ \\
\hline Talidomida & Anlodipino & Moderado & $\begin{array}{l}\text { Pode causar lentidão na } \\
\text { frequência ou ritmo } \\
\text { cardíaco irregular }\end{array}$ & $\begin{array}{l}\text { Necessário ajuste da dose ou } \\
\text { frequente monitoramento da } \\
\text { terapia; uso de alternativas } \\
\text { que não possuam interações }\end{array}$ \\
\hline
\end{tabular}


Research, Society and Development, v. 10, n. 4, e33110413482, 2021

(CC BY 4.0) | ISSN 2525-3409 | DOI: http://dx.doi.org/10.33448/rsd-v10i4.13482

\begin{tabular}{|c|c|c|c|c|}
\hline Hidroxicloroquina & Metotrexato & Leve & $\begin{array}{c}\text { Aumento da incidência } \\
\text { dos efeitos adversos }\end{array}$ & $\begin{array}{l}\text { Não existem estudos que } \\
\text { mostrem interações entre } \\
\text { estes medicamentos }\end{array}$ \\
\hline Cloroquina & Fenitoína & Moderado & $\begin{array}{c}\text { A Cloroquina torna a } \\
\text { Fenitoína menos eficaz } \\
\text { para o tratamento da } \\
\text { epilepsia ou na prevenção } \\
\text { de convulsões }\end{array}$ & $\begin{array}{l}\text { Acompanhamento terapêutico } \\
\text { mais frequente ou prescrição } \\
\text { de medicações que não } \\
\text { interajam }\end{array}$ \\
\hline Cloroquina & Cálcio & Moderado & $\begin{array}{l}\text { Interfere na absorção da } \\
\text { Cloroquina, diminuindo } \\
\text { sua eficácia }\end{array}$ & $\begin{array}{c}\text { Separar a dosagem dos } \\
\text { medicamentos por melo } \\
\text { menos } 4 \text { horas }\end{array}$ \\
\hline Prednisona & Fenitoína & Moderado & $\begin{array}{c}\text { A Fenitoína pode } \\
\text { diminuir a eficácia da } \\
\text { Prednisona }\end{array}$ & $\begin{array}{l}\text { Pode ser necessário dobrar a } \\
\text { dose de Prednisona. A dose } \\
\text { de Fenitoína também pode } \\
\text { precisar ser aumentada. }\end{array}$ \\
\hline Metotrexato & Omeprazol & Grave & $\begin{array}{c}\text { Omeprazol aumenta as } \\
\text { concentrações séricas de } \\
\text { Metotrexato, aumentado o } \\
\text { risco de toxicidade do } \\
\text { mesmo }\end{array}$ & $\begin{array}{c}\text { Interromper uso do } \\
\text { Omeprazol dias antes de } \\
\text { iniciar tratamento com } \\
\text { Metotrexato. Como protetor } \\
\text { gástrico, considera-se a } \\
\text { alternativa de inibidores dos } \\
\text { receptores } \mathrm{H} 2 \text { de Histamina }\end{array}$ \\
\hline Prednisona & Metotrexato & Moderado & $\begin{array}{c}\text { Prednisona pode } \\
\text { aumentar os níveis séricos } \\
\text { ou os efeitos colaterais do } \\
\text { Metotrexato }\end{array}$ & $\begin{array}{l}\text { Necessário ajuste da dose ou } \\
\text { frequente monitoramento da } \\
\text { terapia }\end{array}$ \\
\hline Metotrexato & Paracetamol & Moderado & $\begin{array}{l}\text { Podem causar toxicidade } \\
\text { hematológica e } \\
\text { gastrintestinal severa }\end{array}$ & $\begin{array}{l}\text { Necessário ajuste da dose ou } \\
\text { frequente monitoramento da } \\
\text { terapia. Em baixas doses essa } \\
\text { associação é bem tolerada. }\end{array}$ \\
\hline
\end{tabular}

Fonte: Autores (2019).

No Quadro 6 são apresentados os esquemas terapêuticos que possuem interação farmacológica entre si, utilizados pelos pacientes entrevistados. A prednisona esteve presente em 70\% dos esquemas terapêuticos, apresentando-se também como o medicamento de maior número de interações que envolve a terapia medicamentosa do LES. Entre os medicamentos utilizados pelos pacientes acompanhados, foram selecionados todos que estavam em uso, incluindo os medicamentos da terapia e os externos à terapia, para a avaliação do risco de interação, correspondentes a 22 substâncias ativas diferentes e 13 grupos terapêuticos.

O Gráfico 2 apresenta a frequência percentual dos grupos terapêuticos, utilizados no tratamento do LES. Os grupos terapêuticos que apresentaram maior frequência foram os corticosteroides $(80 \%)$; os antimaláricos $(80 \%)$ e por último foi observado que dois pacientes distintos utilizam os medicamentos das classes imunossupressores (20\%) e antineoplásicos (20\%), além do medicamento talidomida (classe sedativo hipnótico), que se apresentou em menor frequência (10\%). Para esses grupos, os fármacos mais utilizados foram, respectivamente, prednisona (70\%); hidroxicloroquina (70\%); azatioprina (20\%); metotrexato $(20 \%)$ e talidomida $(10 \%)$. 
Gráfico 2 - Grupos terapêuticos mais utilizados pelos pacientes em tratamento do LES.

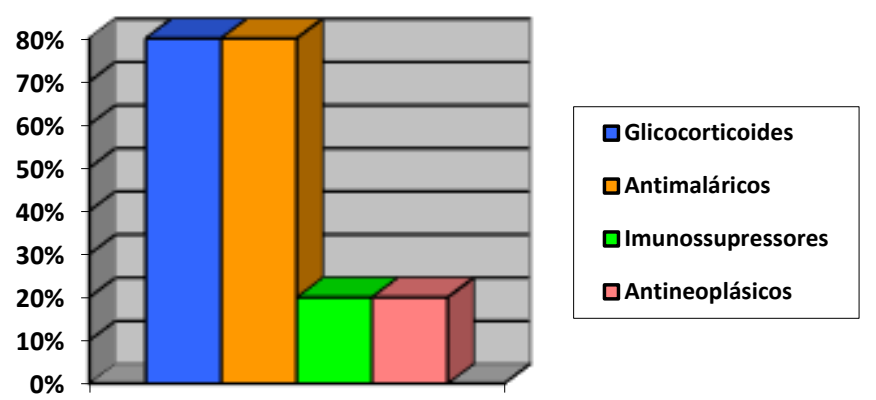

Fonte: Autores (2019).

O tratamento da pessoa com LES depende do tipo de manifestação apresentada e deve, portanto, ser individualizado. Dessa forma, o paciente pode necessitar de um ou mais medicamentos em uma fase (ativa da doença) e poucos ou nenhum em outras fases (não ativas ou em remissão). O tratamento sempre inclui medicamentos para regular as alterações imunológicas do LES e de medicamentos gerais para regular alterações que a pessoa apresente em consequência da inflamação. Os fármacos utilizados que agem na modulação do sistema imunológico no LES incluem os antimaláricos (hidroxicloroquina e cloroquina) e os glicocorticóides (GCs), independentemente do órgão ou sistema afetado pela doença. Os GC apresentam vários efeitos adversos e, com isso, devem ser aplicados na menor dose efetiva para o controle da atividade da doença. Para pacientes em uso de antimaláricos e que não conseguem atingir a dose de manutenção de prednisona, há indicação de associação de outro medicamento, como a Azatioprina (AZA), da classe dos imunossupressores e o metotrexato (MTX), da classe dos antineoplásicos (Ministério da Saúde, 2013).

Foram encontradas 16 interações medicamentosas, na qual 9 destas envolvem a prednisona em associação. Em relação à classificação das potenciais interações identificadas no estudo, 87,5\% foram classificadas como moderadas, 6,25\% apresentaram-se como leve e 6,25\%, como grave. Ao avaliar a adesão à terapêutica Medicamentosa em pacientes com Lúpus eritematoso sistêmico atendidos em Hospital Universitário na cidade do Rio de Janeiro, Santos (2009), descreveu que dos pacientes entrevistados, 55,29\% faziam o uso da polifarmácia; o número médio mínimo de medicamentos foi 4,9, já o máximo de 13. No presente estudo, dos pacientes entrevistados, $50 \%$ fazem uso da polifarmácia; o número médio mínimo de medicamentos utilizados por paciente foi de 2,6 e o número médio máximo de 6,6, no qual este envolve pacientes que fazem o uso de quatro ou mais medicamentos.

A prednisona estava relacionada em $56,25 \%$ ( $n=9$ interações) de todas as interações medicamentosas encontradas neste estudo. Os medicamentos que atuam sobre os níveis séricos de GC aumentando sua toxicidade, normalmente o fazem através da inibição da CYP3A4. A fenitoína, por ser um indutor de CYP3A4, é capaz de diminuir o nível sérico ou atividade de vários GC sistêmicos (Anti, Giorgi \& Chahade, 2008).

Os corticoides são substâncias sintéticas semelhantes aos hormônios produzidos pelas glândulas adrenais, como o cortisol e a aldosterona, que regulam várias funções do organismo. Eles atuam como supressores potentes da inflamação por serem frequentemente usados em uma grande variedade de tratamentos para doenças inflamatórias e autoimunes estão classificados como fármacos mais prescritos para estas patologias (Nunes, 2016).

A intolerância gástrica é um sintoma frequente do uso de GC, inclusive para aqueles que fazem curtos períodos de tratamento. Entretanto, os GC, isoladamente, têm baixa probabilidade de causar hemorragia digestiva ou úlcera péptica. Pacientes que fazem uso de medicamentos dessa classe, em conjunto com anti-inflamatórios não esteroidais (AINES) e anticoagulantes, 
têm risco maior de sangramento digestivo, assim como presença de neoplasia maligna e úlcera digestiva, sendo nestes casos indicado uso de medicamentos profiláticos. Não há, entretanto, estudos suficientes demonstrando o melhor medicamento a ser usado nesses pacientes (bloqueador $\mathrm{H} 2$, inibidor bomba de prótons) e a escolha deve levar em consideração os efeitos colaterais, dosagem e custo (Pereira et al., 2007).

As interações relacionadas ao metotrexato e classificadas como maiores podem resultar em aumento das concentrações plasmáticas de seu metabólito ativo, potencializando seus efeitos adversos, que incluem leucopenia, trombocitopenia, anemia, hepatoxicidade, nefrotoxicidade e ulcerações em mucosas. O metotrexato e os AINEs, em associação, podem causar uma série de complicações, incluindo toxicidade hematológica e gastrintestinal severa (Bagatini et al., 2011).

Conforme Suzuki et al. (2009), a utilização concomitante de metotrexato com omeprazol (interação grave), pode aumentar o risco de toxicidade do primeiro, pois, a coadministração de inibidores da bomba de prótons pode retardar a excreção de metotrexato e potencializar seus efeitos adversos. As concentrações séricas diminuem rapidamente após a interrupção do uso do omeprazol. Por isso, pacientes que utilizam essa associação devem fazer um estrito monitoramento, a fim de evitar possíveis danos resultantes da alta concentração de metotrexato no organismo.

\section{Conclusão}

O presente trabalho visou contribuir para o entendimento dos fatores associados à adesão à terapêutica medicamentosa entre os pacientes com LES. Apesar da carência de estudos comparativos que avaliem a adesão no LES, os resultados do presente estudo permitem concluir que a adesão à terapêutica medicamentosa é um problema complexo e multifatorial e que o seguimento farmacoterapêutico é uma boa alternativa para avaliar a adesão e as RAMs associadas ao tratamento. Dessa forma, recomendase que pacientes com LES contem com equipes multidisciplinares, seguimento farmacoterapêutico e educação em saúde para minimizar ou prevenir o risco potencial de reações adversas e interações medicamentosas. Isso deve contribuir para o uso racional desses medicamentos e para a melhoria das condições de saúde desses pacientes. Novas pesquisas referentes a esse tema podem ser desenvolvidas com o objetivo de quantificar e qualificar a importância do acompanhamento farmacoterapêutico em pacientes portadores de LES.

\section{Referências}

Aizenstein, M. L. \& Tomassi, M. H. (2011). Problemas relacionados a medicamentos; reações adversas a medicamentos e erros de medicação: a necessidade de uma padronização nas definições e classificações. Revista de Ciências Farmacêuticas Básica e Aplicada, 32(2), 169-173. https://rcfba.fcfar.unesp.br/index.php/ojs/article/view/341

Anti, S. M. A., Giorgi, R. D. N. \& Chahade, W. H. (2008). Antiinflamatórios hormonais: glicocorticóides. Einstein, 6(1), 159-165. https://apps.einstein.br/revista/arquivos/PDF/923-Einstein\%20Suplemento\%20v6n1\%20pS159-165.pdf

Araújo, G. B. S., Garcia, T. R. (2006). Adesão ao tratamento anti-hipertensivo: uma análise conceitual. Revista Eletrônica de Enfermagem, 8(2), 259-272. https://www.fen.ufg.br/revista/revista8_2/v8n2a11.htm

Bagatini, F. et al. (2011). Potenciais interações medicamentosas em pacientes com artrite reumatoide. Revista Brasileira de Reumatologia, 51(1), 29-39. https://www.scielo.br/pdf/rbr/v51n1/v51n1a03.pdf

Barreto, M. S. (2014). Prevalência de não adesão à farmacoterapia anti-hipertensiva e fatores associados. Revista Brasileira de Enfermagem, 68(1), 60-67. http://www.scielo.br/pdf/reben/v68n1/0034-7167-reben-68-01-0060.pdf

Ben, A. J., Neumann, C. R. \& Mengue, S. S. (2012). Teste de Morisky-Green e Brief Medication Questionnaire para avaliar adesão a medicamentos. Revista de Saúde Pública, 46(2), 279-289. https://doi.org/10.1590/S0034-89102012005000013

Brasil. (2012). RESOLUÇÃO No 466, DE 12 DE DEZEMBRO DE 2012. Ministério da Saúde - Conselho Nacional de Saúde. http://bvsms.saude.gov.br/bvs/saudelegis/cns/2013/res0466_12_12_2012.html

Borges, J. W. P. et al. (2011). Utilização de questionários validados para mensurar a adesão ao tratamento da hipertensão arterial: uma revisão integ rativa. Revista da Escola de Enfermagem da USP, 46(2), 487-494. http://www.scielo.br/pdf/reeusp/v46n2/a30v46n2.pdf

Brune, M. F. S. S. et al. (2014). O Método Dáder na atenção farmacêutica em pacientes hipertensos no município de Pontal do Araguaia-MT, Brasil. O Mundo da Saúde, 38(4), 402-409. http://www.saocamilo-sp.br/pdf/mundo_saude/155566/A05.pdf 
Cabrera, Z. C. (2016). Adesão de portadores de depressão ao tratamento: uma proposta em estratégia saúde da família. (Especialização, Universidade Federal de Minas Gerais, Brasil). https://www.nescon.medicina.ufmg.br/biblioteca/imagem/zoe-castillo-cabrera.pdf

Campos, A. P. R. et al. (2013). Depressão e qualidade de vida em indivíduos com artrite reumatoide e indivíduos com saúde estável: um estudo comparativo. Revista Fitoterapia e Pesquisa, 20(4), 401-407. http://www.scielo.br/pdf/fp/v20n4/en_v20n4a16.pdf

Cardoso, G. N. (2011). Além da prescrição: a má adesão ao tratamento medicamentoso da hipertensão arterial sistêmica. (Especialização, Universidade Federal de Minas Gerais, Brasil).

Costa, L. M. \& Coimbra, C. C. B. E. (2014). Lúpus Eritematoso Sistêmico: Incidência e tratamento em mulheres. Revista Uningá Review, 20(1), 81-86. https://www.mastereditora.com.br/periodico/20141001_084139.pdf

Costa, C., Santos, J. M. \& Ferreira, C. A. (2003). Lúpus Eritematoso Sistêmico - Episódio terminal de rápida evolução, com choque e falência multiorgânica. Revista Online de Casos Clínicos em Medicina Interna, 10(2). https://www.spmi.pt/revista/vol10/vol10-n2-lupus.pdf

Costa, G. M. A. et al. (2016). Qualidade de vida de pacientes com insuficiência renal crônica em tratamento hemodialítico. Revista Enfermeira Global, 43(2), 73-86. Recuperado de http://scielo.isciii.es/pdf/eg/v15n43/pt_clinica3.pdf

Conde, S. R. S. S. et al. (2009). Estudo Clínico-Epidemiológico de pacientes com Lúpus Eritematoso Sistêmico, em uma população da Amazônia Oriental. Revista Ciência \& Saúde Coletiva, 20(8), 81-86. http://files.bvs.br/upload/S/0101-5907/2009/v23n2/a1998.pdf

Dáder, M. J. F., Hernández, D. S. \& Castro, M.M.S. (2009). Método Dáder: Manual de Seguimento Farmacoterapêutico. (3 ed.). Granada.

Farias, G. \& Martins, R. (2013). Qualidade de vida da pessoa com doença pulmonar obstrutiva crônica. Revista Millenium, 45(2), 195-209. https://revistas.rcaap.pt/millenium/article/view/8102

Ferreira, C. A. L. (2015). Pesquisa quantitativa e qualitativa: perspectivas para o campo da educação. Revista Mosaico, 8(2), 173-182. Recuperado de http://seer.pucgoias.edu.br/index.php/mosaico/article/download/4424/2546

Furlan, L. S. F. et al. (2018). Qualidade de vida em tratamento de lúpus eritematoso sistêmico com antimaláricos. Rev. Soc. Bras. Clin. Med. 16(1), 2-6. http://docs.bvsalud.org/biblioref/2018/06/884974/1612-6.pdf

Freire, E. A. M., Souto, L. M. \& Ciconelli, R. M. (2011). Medidas de avaliação em lúpus eritematoso sistêmico. Revista Brasileira de Reumatologia, 51(1), 7078. http://www.scielo.br/pdf/rbr/v51n1/v51n1a06.pdf

Fritzen, J. S., Motter, F. R. \& Paniz, V. M. V. (2017). Acesso regular e adesão a medicações do componente especializado assistência farmacêutica. Revista de Saúde Pública, 1(6), 51-109. http://www.scielo.br/pdf/rsp/v51/pt_0034-8910-rsp-S1518-87872017051006932.pdf

Galindo, C. V. F. \& Veiga, R. K. A. (2011). Características clínicas e diagnósticas dos lúpus eritematoso sistêmico: uma revisão. Revista Eletrônica de Farmácia, 7(4), 13. https://revistas.ufg.br/REF/article/view/13231/8552

Jacomini, L. C. L. \& Silva, N.A. (2011). Interações medicamentosas: uma contribuição para o uso racional de imunossupressores sintéticos e biológicos. Revista Brasileira de Reumatologia, 51(2). https://www.scielo.br/scielo.php?script=sci_arttext\&pid=S0482-50042011000200006

Marnet, E. B. O. \& Gonçalves, M. (2016). Lúpus Eritematoso Sistêmico em sua Associação com Doenças Psiquiátricas: revisão de literatura. Revista Psychiatry online Brasil, 21(7), 1. https://www.polbr.med.br/ano16/prat0716.php

Meneses, L. L. et al. (2010). Atenção farmacêutica ao idoso: fundamentos e propostas. Geriatrics, Gerontology and Aging, 4(3), 154-161. https://cdn.publisher.gn1.link/ggaging.com/pdf/v4n3a07.pdf

Ministério da Saúde. (2013). PORTARIA N ${ }^{\circ}$ 100, DE 7 DE FEVEREIRO DE 2013. Ministério da Saúde - Secretaria de Atenção à Saúde. http://bvsms.saude.gov.br/bvs/saudelegis/sas/2013/prt0100_07_02_2013.html

Nunes, D. S. (2016). Reações adversas relacionadas ao uso de corticóides em idosos: revisão de literatura. (Trabalho de Conclusão de Curso, Faculdade Maria Milza, Brasil). http://131.0.244.66:8082/jspui/bitstream/123456789/266/1/MONOGRAFIA\%20VERS\%c3\%83O\%20FINAL.pdf

Prado, D. M. et al. (2017). Perfil dos Pacientes Portadores de Lúpus Eritematoso Sistêmico atendidos pelo Componente Especializado da Assistência Farmacêutica em uma Regional de Saúde. Id on Line Revista Multidisciplinar e de Psicologia, 11(38), 808-823. https://idonline.emnuvens.com.br/id/article/view/954/1348

Pereira, A. L. C. et al. (2007). Uso sistêmico de corticosteróides: revisão de literatura. Revista Medicina Cutânea Íbero-Latino-Americana, 35(1), 35-50. http://www.saudedireta.com.br/docsupload/134442634406-091.pdf

Reis, M. G. \& Costa, I. P. (2010). Qualidade de vida de pacientes com lúpus eritematoso sistêmico. Revista Brasileira de Reumatologia, 50(5), 408-422. https://www.scielo.br/scielo.php?pid=S0482-50042013000100008\&script=sci_abstract\&tlng=pt

Rodrigues, D. R. et al. (2017). Diagnóstico clínico e laboratorial do Lúpus Eritematoso Sistêmico. Revista de Patologia do Tocantins, 14(2), 1-6. https://sistemas.uft.edu.br/periodicos/index.php/patologia/article/view/3448/9738

Santos, M. O. (2009). Avaliação da adesão à terapêutica medicamentosa em paciente com Lúpus Eritematoso Sistêmico atendidos em Hospital Universitário na cidade do Rio de Janeiro, Brasil. (Tese de Doutorado, Escola Nacional de Saúde Pública Sérgio Arouca, Brasil). https://www.arca.fiocruz.br/handle/icict/2417

Santos, P. C. J. L. (2016). Atenção farmacêutica: contexto atual, exames laboratoriais e acompanhamento farmacoterapêutico. (1 ed.). Editora Atheneu.

Sousa, M. M., Oliveira, J. S., \& Soares, M. J. G. O. (2017). Qualidade de vida de pacientes com insuficiência cardíaca: revisão integrativa. Revista de Enfermagem UFPE, 11(3), 48-50. https://periodicos.ufpe.br/revistas/revistaenfermagem/article/viewFile/13505/16242 
Research, Society and Development, v. 10, n. 4, e33110413482, 2021

(CC BY 4.0) | ISSN 2525-3409 | DOI: http://dx.doi.org/10.33448/rsd-v10i4.13482

Sousa, E. N., Silva, R. C. \& Partata, A. K. (2011). A importância do farmacêutico no tratamento da depressão pós parto. Revista Infarma, 23(9). http://revistas.cff.org.br/?journal=infarma\&page=article\&op=view\&path $\% 5 \mathrm{~B} \% 5 \mathrm{D}=27 \&$ path $\% 5 \mathrm{~B} \% 5 \mathrm{D}=21$

Suzuki, K. et al. (2009). Co-administration of proton pump inhibitors delays elimination of plasma methotrexate in high-dose methotrexate therapy. $\mathrm{Br} J \mathrm{Clin}$ Pharmacol, 67(1), 44-49. https://pubmed.ncbi.nlm.nih.gov/19076159/

Telles, W.R. et al. (2007). Frequência de Doença Cardiovascular Aterosclerótica e de seus Fatores de Risco em Pacientes com Lúpus Eritematoso Sistêmico. Revista Brasileira de Reumatologia, 47(3), 165-173. https://www.scielo.br/scielo.php?pid=S0482-50042007000300005\&script=sci_abstract\&tlng=pt

Vianna, R. et al. (2010). Lúpus Eritematoso Sistêmico. $\quad$ Revista $\quad$ CicilianaJun, 2(1), 1-3. http://bdm.unb.br/bitstream/10483/1752/1/2001_M\%C3\%A1rcioNascimentodeOliveira.pdf

Zanella, V., \& Assini, F. L. (2008). Identificação de problemas relacionados com medicamentos em pacientes geriátricos na cidade de Concórdia-SC. Revista Brasileira de Farmácia, 89(4), 294-297. 